\title{
Thermophysical Properties and X-Ray Structure Investigations of Polyurethane Ureas, Containing Folate Derivative of Ferrocene
}

\author{
Liudmyla Kulyk*, Rita Rozhnova, Iryna Gladyr, Nataliia Galatenko \\ Department of Polymers of Medical Appointment, Institute of Macromolecular Chemistry of the National Academy of Sciences of Ukraine, \\ Kyiv, Ukraine
}

Email address:

lyudmila.makeeva@gmail.com (L. Kulyk),rozhnovarita@gmail.com (R. Rozhnova), politoks@merlin.net.ua (N. Galatenko)

${ }^{*}$ Corresponding author

\section{To cite this article:}

Liudmyla Kulyk, Rita Rozhnova, Iryna Gladyr, Nataliia Galatenko. Thermophysical Properties and X-Ray Structure Investigations of Polyurethane Ureas, Containing Folate Derivative of Ferrocene American Journal of Physical Chemistry. Vol. 6, No. 4, 2017 , pp. 70-75. doi: 10.11648/j.ajpc.20170604.13

Received: July 1, 2017; Accepted: July 19, 2017; Published: August 15, 2017

\begin{abstract}
Thermophysical properties of polyurethane ureas by thermogravimetry and differential scanning calorimetry were studied. It was shown, that investigated polyurethane ureas are heat-resistant up to $523 \mathrm{~K}$. The structure of polyurethane ureas, containing folate derivative of ferrocene, by wide-angle and small angle X-ray method for different content of folate-derivative of ferrocene was investigated. Wide-angle X-ray diffraction data revealed that the introduction of 0.01 mole of folate derivative of ferrocene to the PUU structure leads to the change of period alternating arrangement of flexible and rigid blocks of PUU, significant degradation of heterogeneous structure, but does not cause changes in amorphous structure of the polymer.
\end{abstract}

Keywords: Polyurethane Urea, Folate Derivative of Ferrocene, Folic Acid, X-Ray Method, Thermogravimetry, Differential Scanning Calorimetry

\section{Introduction}

Development of new bioactive polymer materials, which can stimulate regeneration processes, for treatment of wounds and burns, due to the needs of modern medicine and remains one of the topical areas of polymer chemistry. Polyurethanes are among interesting biomaterials and are being used in a variety of implantable medical devices [1]. Polyurethane is a biocompatible polymer with low cytotoxicity which makes it the potential candidate for applications in tissue engineering, material science, and drug delivery [2]. For example, new rapid hemostatic and mild polyurethane-urea foam (PUUF) wound dressing was prepared by the particle leaching method and vacuum freeze-drying method using 4,4-Methylenebis (cyclohexyl isocyanate), 4,4diaminodicyclohexylmethane and poly (ethylene glycol) as raw materials. This porous PUUF represents a novel mild wound dressing with excellent water uptake, hemostatic effect and low toxicity, and it can promote wound healing and enhance re-epithelialization [3].

As medical supplies this type noteworthy polyurethane ureas (PUU), chemically modified by folate derivative of ferrocene (FKF), which are capable to stimulate tissue regeneration in the place of usage [4]. A set of physical, mechanical, and biomedical research of modified PUU was conducted. It was established, that obtained polymers are non-toxic, biologically stable up to 180 days in terms of the model biological environment 199, and exhibit biological activity by activating of growth and differentiation of fibroblastic elements of the connective tissue [5]. It is interesting to study the processes of structure formation of polymer materials, chemically modified by different amounts of folate derivative of ferrocene, and they thermal properties.

Thus, the aim of this work was to study the characteristics of heterogeneous structure of PUU samples containing different amounts of FKF by wide-angle and small angle Xray methods. 


\section{Methods and Materials}

Polymer film materials in the research were received by synthesis of diisocyanate prepolymer (DP) based on polyoxypropylene glycol (MW 1000) i 2, 4-; 2, 6-toluene diisocyanate, and then the macro chain extending by 4,4 'diaminodiphenylmethane (DADPh) and chemically modifing by folate derivative of ferrocene (FKF) in amount of 0.01 , 0.03, 0.06 i $0.09 \mathrm{~mol}$, named PUU (DADPh)-1, PUU (DADPh)-3, PUU (DADPh)-6, PUU (DADPh)-9 respectively [4]. Initial nonmodifyed sample is called PUU (DADPh).

Thermophysical properties of synthesized PUU were studied by thermogravimetry (TGA) using Q50 device (TA Instruments, USA) in air at temperatures from ambient to +973 K, heating rate $20 \mathrm{deg} / \mathrm{min}$, and by differential scanning calorimetry (DSC) using Q2000 device (TA Instruments, USA) in air at temperatures from 183 to $473 \mathrm{~K}$, heating rate $20 \mathrm{deg} / \mathrm{min}$.

Studies of near ordering in the broadcast space fragments of PUU macro chains, containing folate derivative of ferrocene in its composition, performed by wide-angle X-ray scattering (WAXR) using a diffractometer DRON-4-0, which X-ray optical circuit is made "in passing" primary beam of X-rays through the sample. Features of structure of PUU samples heterogeneity were studied by small-angle X-ray scattering (SAXR) using MRC-1 camera, which used crevice beam collimation of the primary X-ray by $\mathrm{O}$. Kratky method. Geometric camera settings satisfy conditions of "infinite" height of collimation gap [6]. Intensity profiles obtained experimentally relatively wide and small angle X-ray scattering were normalized to the value of the investigated PUU sample scattering volume and caused by its primary beam radiation weakening factor. All radiographic studies were performed in $\mathrm{Cu} K_{\alpha}$ anode X-ray tube radiation (wavelength $\lambda=0,154 \mathrm{~nm}$ ) monochromotysed by Ni-filter, at $T=(293 \pm 2) \mathrm{K}$.

\section{Results and Discussion}

Since synthesized PUU are polymer materials for medical application, which require sterilization of heat before use, there is a need to study the thermal properties of received materials.

Temperature of the beginning of decomposition of PUU samples is in the range from 550 to $567 \mathrm{~K}$ (Figure 1) and is accompanied by a small weight loss (1.15 - 3.95\%). Decomposition of samples occurs at a temperature of maximum decomposition rate in the range from 629 to 652
K. For a number of polymers gradual decline of temperature of maximum decomposition rate with increasing of FKF content in PUU is inherent. PUU samples are heat-resistant up to $523 \mathrm{~K}$, which makes possible dry sterilization usage for these polymeric materials without changing of their characteristics.

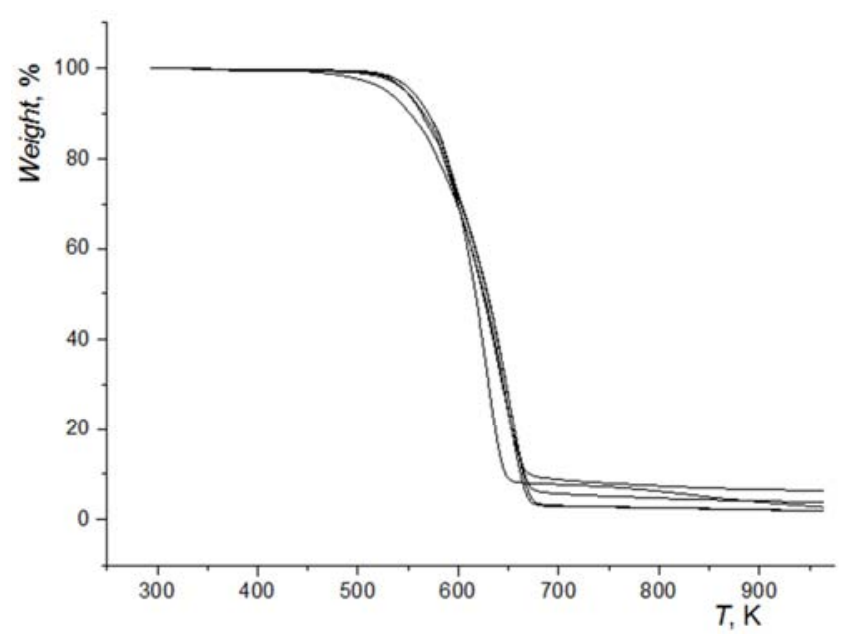

Figure 1. TGA studies of samples PUU (DADPh) (1), PUU (DADPh)-1 (2), PUU (DADPh)-3 (3), PUU (DADPh)-6 (4), PUU(DADPh)-9 (5).

According to DSC data, one temperature switch and, accordingly, one glass transition temperature $(T g)$ on thermograms of PUU without FKF, and chemically modified by FKF, are observed. Thus indicates a one-phase (amorphous) macrostructure of investigated polyurethane ureas. $T g$ of all PUU samples is in the range from 241 to 247 $\mathrm{K}$ (Figure 2).

Heat capacity jump $(\Delta \mathrm{Cp})$ and $T g$ dependence on FKF amount was investigated (Table 1).

Table 1. Thermophysical properties of PUU, chemically modified by FKF.

\begin{tabular}{lll}
\hline Samples & Tg, K & $\Delta \mathbf{C p}, \mathbf{J}$ \\
\hline PUU (DADPh) & 241 & 0.43 \\
PUU (DADPh)-1 & 243 & 0.43 \\
PUU (DADPh)-3 & 243 & 0.42 \\
PUU (DADPh)-6 & 242 & 0.40 \\
PUU (DADPh)-9 & 247 & 0.38 \\
\hline
\end{tabular}

With FKF content increasing in PUU samples $T g$ value slightly increases, and $\Delta \mathrm{Cp}$ gradually decreases.

If consider $\Delta \mathrm{Cp}$ as the measure of the free volume of the system, then the decrease of $\Delta \mathrm{Cp}$ is probably due to the gradual decrease in the free volume in the systems by increasing intermolecular interactions between the fragments containing FKF. 


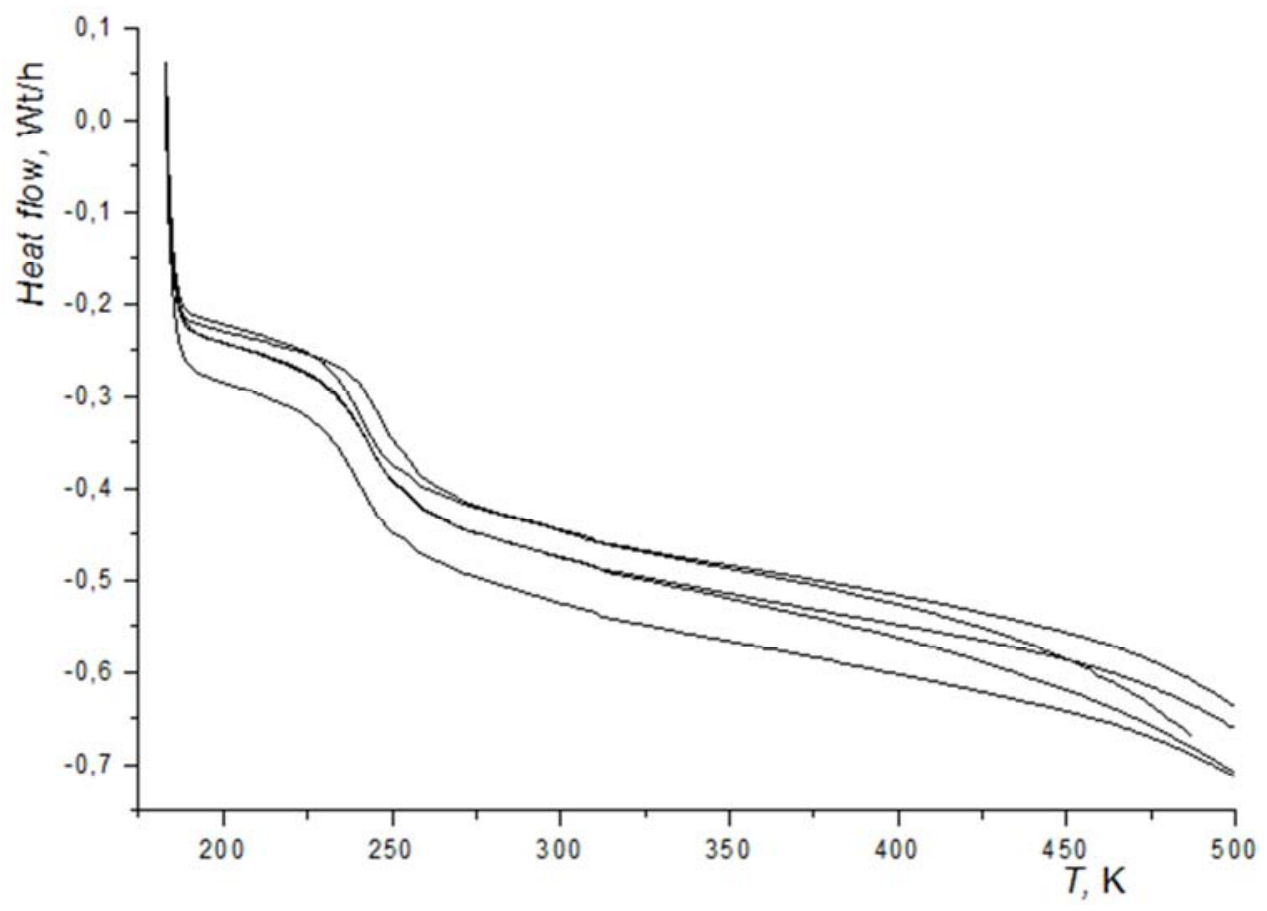

Figure 2. DSC studies of samples PUU (DADPh) (1), PUU (DADPh)-1 (2), PUU (DADPh)-3 (3), PUU (DADPh)-6 (4), PUU (DADPh)-9 (5).

Comparing the wide-angle X-ray diffraction pattern of the initial sample and chemically modified by folate derivative of ferrocene polyurethane ureas samples (Figure 3) it was found, that even a small amount of FKF $(0.01 \mathrm{~mol})$ in PUU composition has an impact on neighbor arrangement features of polymer macro chains fragments. Thus, expression of intense diffraction diffuse (given the significant semiwidth corner) type peak to X-ray diffraction pattern of initial PUU (DADPh) indicates the passing of ordering pieces of macro chains in its volume. Proceeding from the angular position $\left(2 \theta_{m}\right)$ of the diffraction peak maximum $\left(2 \theta_{m} \approx 20,0^{\circ}\right)$ the range order period value $d$ when placing pieces of macro chains in volume of PUU (DADPh) (the average distance between the nearest macro chains fragments), according to the Bragg equation:

$$
d=\lambda\left(2 \sin \theta_{m}\right)^{-1}
$$

\section{is $0,44 \mathrm{~nm}$.}

Attention is drawn to the fact, that the introduction of the smallest quantities $(0.01 \mathrm{~mol})$ of FKF into PUU composition (PUU sample PUU (DADPh)-1), leads to diffraction peak intensity of the diffuse type (an amorphous halo) slightly decreasing without changing of its shape and angular position compared to intensity and magnitude $2 \theta_{m}$ diffraction peak at diffraction PUU (DADPh) (Figure 3, curves 1, 2). Further chemical modification of PUU macro chains by 0.03 and $0.06 \mathrm{~mol}$ of FKF also does not cause changes in the shape and angular position of amorphous halo, but only leads to the gradual decrease in its intensity, compared with the same diffraction peaks at diffraction of PUU (DADPh) and PUU (DADPh)-1 (curves 1-4). This indicates the dominant contribution of DP and confirm the particularly amorphous structure of PUU.

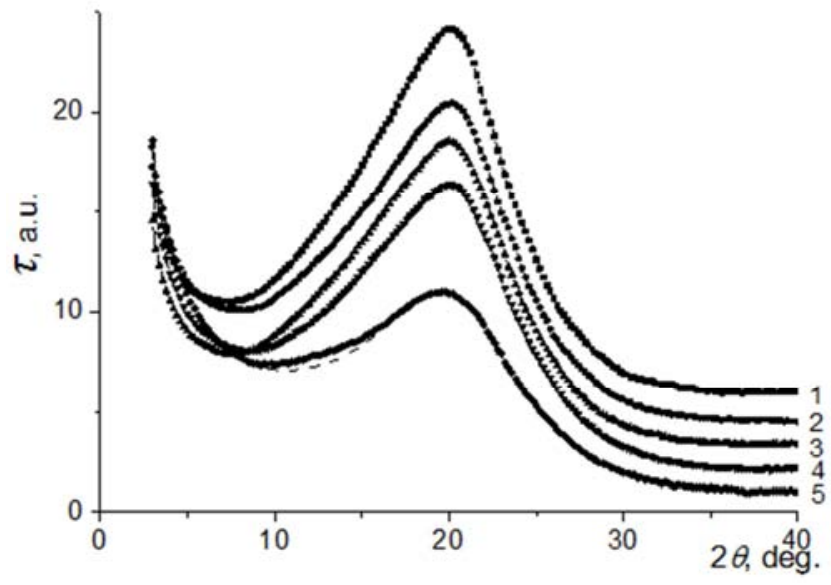

Figure 3. Wide-angle X-ray diffraction of samples PUU (DADPh) (1), PUU (DADPh)-1 (2), PUU (DADPh)-3 (3), PUU (DADPh)-6 (4), PUU (DADPh)9 (5).

However, the increasing of FKF amount $(0.09 \mathrm{~mol})$ in the PUU composition (sample PUU (DADPh)-9) causes significant changes in the diffraction pattern (Figure 3, curve 5) compared to the diffraction pattern of PUU samples PUU (DADPh), PUU (DADPh) -1, PUU (DADPh)-3 and PUU (DADPh)-6 (curves 1-4). In particular, there is the change in the shape and angular position of amorphous halo $\left(2 \theta_{m} \approx\right.$ 19.6) characterizing somewhat greater distance between adjacent FKF-containing fragments of macro chains $(d=$ $0.45 \mathrm{~nm}$ ) in volume of PUU (DADPh)-9 compared to samples chemically modified by fewer amount of FKF (0.01-0.06 mol). In addition, the X-ray diffraction of PUU (DADPh)-9 also takes place almost linear change in the intensity of X-ray scattering angles $(2 \theta)$ from $9^{\circ}$ to $14^{\circ}$ (dashed line indicates progress in the absence of diffraction 
in this angular field of linear change in intensity) that describes the expression of other structural effects than amorphous structure PUU samples, chemically modified by FKF. As you know, in $2 \theta$ from $10^{\circ}$ to $12^{\circ}$ is a manifestation of the amorphous halo peak, which characterizes the near streamlining fragments of macro chains of the polymers that are complex related by cations of two and trivalent metals [79]. Change the intensity of X-ray scattering to diffraction PUU (DADPh)-9 may be a manifestation of complex action of iron cations of FKF against the harsh polar groups of PUU blocks.

Thus, on the basis of wide-angle X-ray diffraction revealed that, increasing of the concentration of FKF from 0.01 to 0.06 mol in PUU composition does not cause changes in amorphous structure of the polymer, which is similar to the structure of the initial sample of polyurethane urea (PUU $(\mathrm{DADPh})$ ). The gradual decreasing of the intensity of amorphous halo at diffractograms of samples PUU (DADPh) -1, PUU (DADPh) -3 and PUU (DADPh)-6 with increasing of content of FKF in their composition, may be associated with decreasing in the macro chains of polymers appropriate number of moles $(0.01-0.09)$ and fragments of DP and DADPh. The concentration of $0.09 \mathrm{~mol}$ of FKF in PUU composition is critical, resulting in a change in the structure of amorphous polymer with a tendency to increasing during the period of near ordering broadcast fragments in space and its macro chains probably is a manifestation of complex formation between atoms of $\mathrm{Fe}$, contained in the molecules of FKF, and polar groups of PUU rigid blocks.

According to the revealed amorphous structure of PUU depending on the content of FKF amount in their composition, it is important to examine the heterogeneous structure of PUU samples. The analysis of the small-angle Xray scattering intensity profiles shows (Figure 4, insert picture shown on the intensity profile of the PUU (DADPh) sample) that the increasing of FKF concentration in PUU composition has a significant impact on their heterogeneous nanosized structure. Thus, the manifestation of low-intensity interference maximum at $2 \theta_{m} \approx 0,98^{\circ}$ at intensity profile of the initial PUU sample (Figure 4, curve 1 and insert picture), indicates the presence in the volume of the polymer of, at least, two types of heterogeneity regions, such as hard domains, and regions enriched by flexible blocks (flexible domains) which placing in space (in volume of PUU $(\mathrm{DADPh})$ is periodic. The period $D$ of rotation in the space of rigid or flexible domains of PUU (DADPh), according to the Bragg equation (1) is $8.6 \mathrm{~nm}$.

Estimation of effective size (order of magnitude) of rigid (flexible) domains was performed by method [9] determining the structural parameter known as inhomogeneity range $l_{p}$ [10]. This parameter has a direct link with an average diameter of both types of heterogeneity regions $\left(<l_{1}>\right.$ and $<l_{2}>$ ) in two-phase system:

$$
l_{p}=\varphi_{2}<l_{1}>=\varphi_{1}<l_{2}>
$$

where $\varphi_{1}, \varphi_{2}-$ volumetric proportion of two types of heterogeneity regions $\left(\varphi_{1}+\varphi_{2}=1\right)$, namely $<l_{1}>$ and $<l_{2}>$ have a greater value than $l_{p}$.

The calculations showed that for PUU(DADPh) value $l_{p}$ $\approx 5,8 \mathrm{~nm}$.

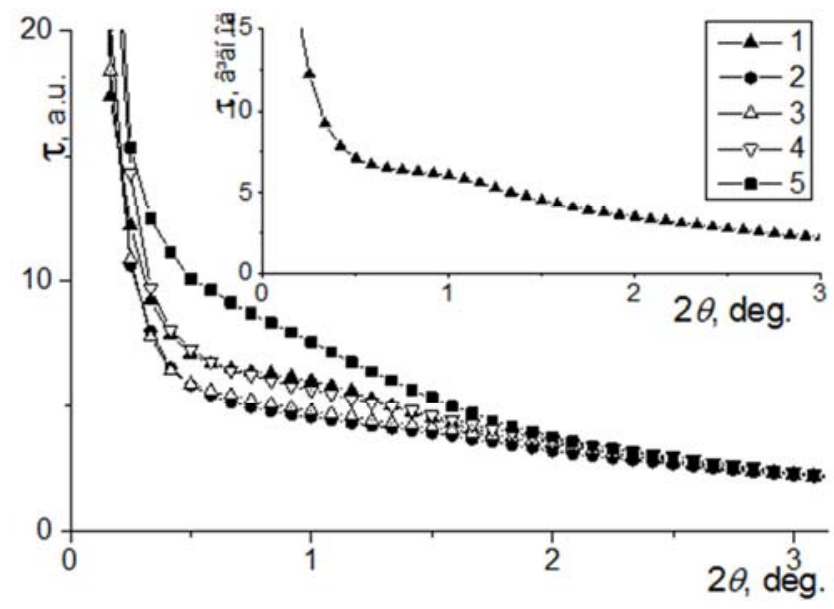

Figure 4. Small-angle X-ray scattering of samples PUU(DADPh) (1), PUU (DADPh)-1 (2), PUU(DADPh)-3 (3), PUU(DADPh)-6 (4), PUU (DADPh)-9 (5).

Table 2. Options of heterogeneous structure of PUU, chemically modified by FKF.

\begin{tabular}{lll}
\hline Samples & $\boldsymbol{l}_{\mathbf{p}}, \boldsymbol{n} \boldsymbol{m}$ & $\boldsymbol{Q}^{\prime}, \boldsymbol{a} \cdot \boldsymbol{u}$. \\
\hline PUU(DADPh) & 5.8 & 1.37 \\
PUU(DADPh)-1 & 7.0 & 1.27 \\
PUU(DADPh)-3 & 4.1 & 1.34 \\
PUU(DADPh)-6 & 3.3 & 1.41 \\
PUU(DADPh)-9 & 5.7 & 1.49 \\
\hline
\end{tabular}

Importantly, that the presence of even $0.01 \mathrm{~mol}$ of FKF in PUU composition causes decreasing of the intensity of smallangle X-ray scattering of sample PUU (DADPh)-1, compared to the intensity that has sample PUU (DADPh), while at the profile of sample PUU (DADPh)-1 there is no interference maximum (Figure 4, curves 1, 2). However, increasing of the concentration of FKF in PUU composition to 0.03 mol leads to the intensity of small-angle X-ray scattering sample PUU (DADPh)-3 slightly increasing, compared to the intensity of sample PUU (DADPh)-1, while linear decline in their intensity profiles is stored (Figure 4, curves 2, 3). Much more growth of scattering intensity observed by content of 0.06 mol of FKF in PUU composition, with the same intensity profile form with those, that take place at profiles PUU (DADPh)-1 and PUU (DADPh)-3, and, especially significant scattering intensity has PUU, chemically modified by 0.09 mol of FKF (Figure 4, curves 4, 5). Analysis of the intensity profiles of samples PUU (DADPh)-1, PUU (DADPh)-3, PUU (DADPh)-6, PUU (DADPh)-9, presented in the form of dependence $\tilde{I}$ from $2 \theta$ (Figure 4 ) and $s^{3} \tilde{I}$ (s) from $s^{3}$ (Rudand charts [9]), where $\tilde{I}$ - scattering intensity at slit beam collimation of the primary X-rays, $s-$ the magnitude of the wave vector in space of inverse lattice $\left(s=\lambda^{-1} 2 \sin \theta\right)$, revealed, that all PUU samples have heterogeneous structure at nanoscale level. Absence of interference peak at intensity profiles of PUU samples, chemically modified by FKF, is a 
manifestation of the disordered placement in space of the same type (in size electron density) regions of heterogeneity (rigid or flexible domains). However, much less intensity of small-angle X-ray scattering of the sample PUU (DADPh)-1, compared to the intensity of the sample PUU (DADPh), is the result of much lower contrast of the electron density $\Delta \rho$ in its volume $(\Delta \rho=\rho-\langle\rho>$, where $\rho$ and $\langle\rho\rangle-$ local and average value of the electron density in the two-phase system). This can be observed only when in the volume of PUU (DADPh)1 "pseudo three-phase system" morphology is available, for example, with the existence of crystallites of flexible units in the volume of segmented polyurethane [11], ie, when the electron density is changing sequentially at the transition from one to another "pseudo phase". The third one "pseudo phase" in the volume of PUU (DADPh)-1, is, probably, formed by heterogeneity areas, containing rigid blocks, which have complex bounds with iron atoms, which are available in molecules of FKF. This was stated above, in the analysis of wide-angle X-ray diffraction of PUU (DADPh)-9, but, these areas of heterogeneity are available in the volume of all FKF-containing PUU samples. With the increasing of the amount of FKF as part of PUU macro chains, the number of indicated heterogeneity areas is growing, which increases the value of $\Delta \rho$, which is especially true for samples PUU (DADPh)-6 and PUU (DADPh)-9. The results show, that $l_{p}$, with the gradual growth of FKF amount in PUU composition from 0.01 to $0.09 \mathrm{~mol}$, changing of the effective size of heterogeneity areas has extreme character, reaching the lowest value in volume PUU (DADPh)-6 (Table 2).

To determine the relative heterogeneity of PUU samples structure, such structural parameter as Porod invariant $Q^{\prime}$ was evaluated (in the case of slit collimation of X-rays primary beam) [7], which value is independent of the form of heterogeneity areas:

$$
Q^{\prime}=\int_{0}^{\infty} q \tilde{I}(q) d q
$$

where $q=2 \pi s$.

This parameter characterizes the integrated intensity of $\mathrm{X}$ ray scattering by two-phase system and has a direct connection to the fluctuations rms of the electron density $\left(<\Delta \rho^{2}>\right)$ in its volume:

$$
Q^{\prime} \propto<\Delta \rho^{2}>
$$

with $<\Delta \rho^{2}>=\varphi_{1} \varphi_{2}\left(\rho_{1}-\rho_{2}\right)^{2}$, where $\rho_{1}, \rho_{2}-$ electron density of heterogeneity regions $(<>, \propto-$ signs of averaging and direct proportionality, respectively). The calculations show, that $0.01 \mathrm{~mol}$ of FKF leading in PUU composition (PUU (DADPh)-1) causes significant reduction of heterogeneity of structure, compared to the corresponding value of the initial sample PUU (DADPh), while the gradual increasing of the amount of FKF in the volume of PUU samples leads to the linear increasing of the heterogeneity range of their structure.

\section{Conclusion}

In the result of studying the thermophysical properties of polyurethane ureas of chemically modified by folate ferrocene derivative, it was found that the ferrocene derivatives in the polymer structure do not have a significant effect on their thermophysical properties. Synthesized PUU are heat-resistant up to $523 \mathrm{~K}$, which will allow them to be subjected to thermal sterilization in the future. According to the DSC, an insignificant increase in the glass transition temperature and a decrease of $\Delta \mathrm{Cp}$ with an increase in the FKF content in the structure of the PUU can be associated with a decrease in free volume due to an increase in the intermolecular interactions between the FKF-containing fragments of urea polyurethane.

According to the results of radiographic studies, diffraction pattern of all the samples are characterized by one interference maximum, investigated polyurethane ureas have linear structure. At diffractograms is presented one diffuse type diffraction maximum (amorphous halo), indicating that a small percentage of branches may be present, which lies outside of the sensitivity of the device and, probably, is 1$2 \%$. Past studies have found a significant effect of microstructure of FKF molecular chains, especially of iron atoms presence in them, the processes of structure formation in chemically modified by folate-derivative of ferrocene PUU samples. It is shown, that the introduction into PUU rigid blocks composition only $0.01 \mathrm{~mol}$ of FKF causes significant degradation of heterogeneous structure, compared to PUU (DADPh), while their amorphous structure is almost the same. Based on this an assumption was made about formation, in addition to rigid and flexible domains, heterogeneity regions of the fragments of rigid blocks, which polar groups formed complex bonds with the atoms of iron. As the result, formed "psevdo three-phase" structure with significantly less contrast of electron density in the PUU (DADPh) -1 composition, compared to PUU (DADPh). This "psevdo three-phase" morphology is typical for all PUU samples, chemically modified by FKF, but at 0.06 and 0.09 mol concentrations of FKF in PUU, heterogeneity regions formed by fragments of rigid blocks, which are bounded in complex by iron atoms of FKF, formed relatively large value of the electron density contrast $\Delta \rho$ with flexible domains, resulting in high heterogeneity structure of samples PUU (DADPh) -6 and PUU (DADPh)-9.

\section{References}

[1] J. Zhang, T. M. Woodruff, R. J. Clark, D. J. Martin, R. F.: Acta Biomaterialia, 2016, 41, 264.

[2] M. Alishiri, A. Shojaei, M. J. Abdekhodaie, R. Adhikari, P. A. Gunatillake, I. Griffiths, et al: RSC Adv., 2016, 6, 8743.

[3] Xiangyu Liu, Yuqing Niu, Kevin C. Chen, Shiguo Chen: Materials Science and Engineering: C, 2017, 71, 289.

[4] Makeeva L., Gladyr I, Rozhnova R., Galatenko N.: Polimernyj Journal, 2014, 36, № 2, 181. 
[5] Galatenko N., Rozhnova R., Kulyk L., Kulyesh D.: Chemistry, Physics and Technology of Surface, 2016, 7, № 3, 344.

[6] Kratky O., Pilz I., Schmitz P.: Journal of Colloid and Interface Science, 1966, 21, N1, 24.

[7] Shtompel V., Kercha Yu.: Structure linejnych polyurethanov. Naukova dumka, Kiev, 2008.

[8] Demchenko V., Shtompel V., Ryabov S.: Vysokomolekulyarnye Soedinenia. Seria B, 2015, 57, № 5, 466.
[9] Perret R., Ruland W.: Kolloid-Zeitschrift und Zeitschrift für Polymere, 1971, 247, Heft 1-2, 835.

[10] Guinier A., Fourner G.: Small-angle scattering of x-rays. John Wiley \& Sons, Inc., New York, 1955.

[11] Vilenskij V., Kercha Yu, Shtompel V., Hasanov R.: Vysokomolekulyarnye Soedinenia. Seria A, 1987, 29, № 7, 1377. 